\title{
Body mass index cutoff points for evaluation of nutritional status in Brazilian children and adolescents
}

\author{
Valores críticos do índice de massa corporal para classificação \\ do estado nutricional de crianças e adolescentes brasileiros
}

\author{
Wolney L. Conde ${ }^{1}$, Carlos A. Monteiro ${ }^{2}$
}

\section{Resumo}

Objetivo: Delinear sistema de classificação, composto de curva de referência e valores críticos, baseado na distribuição do índice de massa corporal (IMC) em população de referência nacional e destinado à avaliação do estado nutricional de crianças e adolescentes brasileiros.

Método: Dados de 13.279 homens e 12.823 mulheres com idade de 2 a 19 anos, extraídos da Pesquisa Nacional Nutrição e Saúde (1989), foram utilizados para construir a curva de referência. Utilizou-se o método LMS para o cálculo dos parâmetros da curva do IMC e a função polinomial para modelar esses parâmetros ao longo das idades. Os valores críticos para classificação do estado nutricional em déficit de peso, excesso de peso e obesidade foram expressos de acordo com centis e valores do IMC equivalentes a $17,5,25$ e $30 \mathrm{~kg} / \mathrm{m}^{2}$, respectivamente, aos 20 anos.

Resultados: Os valores dos parâmetros $\mathrm{L}, \mathrm{M}$ e $\mathrm{S}$ foram tabulados em intervalos semestrais para cada sexo. A partir desses valores, foi construído o gráfico com nove centis da distribuição de referência do IMC. Foram apresentados os valores críticos equivalentes aos valores de $17,5,25$ e $30 \mathrm{~kg} / \mathrm{m}^{2}$ no início da idade adulta.

Conclusão: $O$ sistema classificatório apresentado pode ser utilizado em avaliações clínicas e epidemiológicas, é similar metodologicamente à grande parte das curvas nacionais já apresentadas e oferece, ainda, a definição de baixo peso.

J Pediatr (Rio J). 2006;82(4):266-72: Padrão de referência, estado nutricional, crianças, adolescentes, IMC.

\section{Introdução}

O uso de medidas antropométricas na avaliação do estado nutricional tem se tornado, embora com limitações, o modo mais prático e de menor custo para análise de indivíduos e populações, seja em ações clínicas, de triagem, ou mesmo em monitoração de tendências.

Um padrão ou curva de referência antropométrica é a representação sumarizada da distribuição de determinada medida antropométrica segundo uma co-variável (usual-

1. Professor Doutor, Universidade de São Paulo (USP), São Paulo, SP.

2. Professor titular, USP, São Paulo, SP.

Artigo submetido em 27.09.05, aceito em 29.04.06.

Como citar este artigo: Conde WL, Monteiro CA. Body mass index cutoff points for evaluation of nutritional status in Brazilian children and adolescents. J Pediatr (Rio J). 2006;82:266-72.

\section{Abstract}

Objective: To delineate a classification system, comprising reference curves and cutoff points, based on the distribution of body mass index (BMI) across a national reference population and designed for the assessment of the nutritional status of Brazilian children and adolescents.

Methods: Data from 13,279 males and 12,823 females aged from 2 to 19 years, extracted from the National Nutrition and Health Survey dataset (1989), were used to construct a reference curve. The LMS method was employed to calculate the BMI curve parameters and polynomial functions were used to model these parameters against age. The cutoff values for classifying nutritional status as underweight, overweight and obese were expressed as centiles and BMI values equivalent to $17.5,25$ and $30 \mathrm{~kg} / \mathrm{m}^{2}$ at 20 years, respectively.

Results: Values for the $\mathrm{L}, \mathrm{M}$ and $\mathrm{S}$ parameters were tabulated at 6-month intervals for each sex. Using these values, a graph was plotted with nine BMI distribution reference centiles. Cutoff values were presented that are equivalent to BMIs of $17.5,25$ and $30 \mathrm{~kg} / \mathrm{m}^{2}$ at the start of adulthood.

Conclusions: The classification system presented here can be used for clinical and epidemiological assessments, it is methodologically similar to the majority of national curves that have been presented to date and, furthermore, it offers a definition of underweight.

J Pediatr (Rio J). 2006;82(4):266-72: Reference standard, nutritional status, children, adolescents, BMI.

mente, idade) em cada sexo ${ }^{1}$. As curvas de referência representam o "modelo empírico saudável" e servem, a um só tempo, para classificar (comparar com grupo de referência) e para diagnosticar (separar indivíduos saudáveis dos não-saudáveis) o estado nutricional de um indivíduo ou população.

A fundamentação do diagnóstico antropométrico do estado nutricional infantil vem oscilando, historicamente, entre bases epidemiológicas e estatísticas. Entre os primeiros sistemas classificatórios utilizados estão aqueles propostos por Gomez, McLaren e Waterlow. Essas propostas debatiam-se em torno dos indicadores e desfechos selecionados para descrever e classificar o estado nutricional infantil $^{2}$. Waterlow, dentre os citados, é o primeiro a propor um sistema classificatório efetivamente probabilístico - isto é, estatístico - para lidar com a classificação do estado 
nutricional infantil. Essa proposta foi adotada pela OMS em $1975^{3}$ e, desde então, tem predominado no diagnóstico do estado nutricional infantil. Todavia, a opção pela classificação probabilística no caso do estado nutricional infantil contrasta com a classificação do estado nutricional em adultos a partir do índice de massa corporal (IMC). Neste caso, o sistema classificatório está baseado no risco de mortalidade ou doença associado a diferentes intervalos do IMC, um critério epidemiológico.

O estado nutricional dos menores de 20 anos tradicionalmente vem sendo classificado com base em critérios probabilísticos. Em 1997, a International Obesity Task Force (IOTF) propôs definir o estado nutricional dos menores de 20 anos com base em desfechos que, na idade adulta, definiam os diagnósticos de desnutrição, excesso de peso e obesidade e/ou na alteração de diversos indicadores bioquímicos associados a doenças crônicas na fase adulta ${ }^{4}$. Em 2000, foi publicado o conjunto de valores críticos para definir excesso de peso e obesidade no grupo etário de 2 a 18 anos $^{5}$

O uso das curvas baseadas no IMC para idade para definição do estado nutricional em crianças e adolescentes propiciou, por um lado, soluções práticas e, por outro lado, debates sobre o uso dessas curvas para avaliação do estado nutricional de grupos em crescimento. Os principais pontos em debate são: 1) a natureza universal ou particular da composição corporal, aspecto refletido no debate sobre a adoção de curva de referência local ou internacional 6,$7 ; 2$ ) os fundamentos e propriedades do sistema classificatório baseado no IMC para idade, o que se reflete no debate sobre o uso de critérios estatísticos ou epidemiológicos ${ }^{8}$ e; 3) a influência da maturação sexual sobre a composição corporal e a necessidade de levar em conta ou não o estágio de maturação sexual do avaliado9,10.

O objetivo deste trabalho é apresentar um sistema de referência, baseado no IMC, para avaliação do estado nutricional de crianças e adolescentes brasileiros incluindo o delineamento de uma curva de referência e o estabelecimento de valores críticos "estatísticos" e "funcionais" para o diagnóstico da desnutrição, do excesso de peso e da obesidade.

\section{Material e métodos}

O conjunto de dados utilizado no delineamento da curva nacional de referência do IMC é originário da Pesquisa Nacional Saúde e Nutrição (PNSN) realizada pelo Instituto Brasileiro de Geografia e Estatística em 198911. A preparação rigorosa e o controle de qualidade observados na coleta das medidas antropométricas, além da representatividade nacional (exceto área rural da Região Norte), foram decisivos na escolha dessa base de dados.

Para determinar a adequação dos dados da PNSN aos propósitos deste trabalho, foi realizada a seguinte análise: os dados da PNSN e do Estudo Nacional de Despesa Familiar (realizado 14,5 anos antes) foram padronizados por sexo e idade segundo valores de referência CDC 2000 para altura e peso e, a seguir, comparados. Foram observadas as tendências seculares dos valores de altura para idade inferiores a -2 escores $z$ e dos valores de peso para idade maiores que 2 escores $z$. No período, houve redução no déficit de altura $(-59,5 \%)$ e aumento no excesso de peso $(106,5 \%)$. A freqüência de indivíduos com valores de peso para idade superiores a 2 escores z na PNSN, no entanto, não ultrapassou o valor probabilístico da classificação.

Para este estudo, foram selecionados indivíduos entre 2 e 19 anos e dados completos sobre sexo, idade (expressa em mês), altura (expressa em cm, com uma casa decimal) e peso (expresso em kg, com uma casa decimal). A amostra ficou composta por 13.279 homens e 12.823 mulheres, representando $99 \%$ dos indivíduos disponíveis em cada sexo.

Foram excluídos os valores do IMC inferiores ou superiores a 4 desvios padrão em relação à média, segundo idade e sexo. 0 valor não convencional de \pm 4 desvios foi escolhido para preservar, ao máximo, a heterogeneidade da amostra.

O método utilizado na construção da curva brasileira foi basicamente o mesmo utilizado na construção do padrão internacional do IMC ${ }^{5}$. Em essência, o método LMS assume que, para dados independentes com valores positivos, a transformação Box-Cox idade-específica pode ser empregada para torná-los normalmente distribuídos; os valores $L$, M e S são natural cubic splines com knots em cada intervalo etário ${ }^{12}$. A amostra, em cada sexo, foi separada em faixas etárias de 3 meses. Essa subdivisão trimestral objetivou representar a diversidade da velocidade de crescimento e somar, em cada estrato, 100 ou mais indivíduos, número considerado o mínimo adequado para o método LMS. Em cada estrato, foram calculados os parâmetros L, M e S. O parâmetro $M$ expressa o valor mediano do índice observado no interior de cada estrato; o parâmetro $\mathrm{S}$ representa o coeficiente de variação de cada estrato e o parâmetro $L$, o coeficiente (Box-Cox) empregado para a transformação matemática dos valores do IMC com o objetivo de obter distribuição normal em cada estrato. O valor selecionado para o coeficiente $L$ é aquele cuja transformação produza a menor soma dos quadrados dos desvios da variável. A seguir, as curvas de cada parâmetro foram suavizadas pelo uso de polinômios em cada sexo. Finalmente, por interpolação baseada na média aritmética, foram obtidos os valores mensais da distribuição.

Com esses três parâmetros, torna-se possível construir a curva referente a qualquer centil desejado com o emprego da fórmula:

$$
C_{100 \alpha(t)}=M_{(t)}\left[1+L_{(t)} S_{(t)} Z_{\alpha}\right]_{(t)}^{1 / L_{(t)}}
$$

na qual $Z_{\alpha}$ é o desvio normal equivalente para a área $\alpha$; $C_{100 \alpha(t)}$ é o centil correspondente ao $Z \alpha ; t$ é a idade em meses e $L_{(t)}, M_{(t)}, S_{(t)}$ e $C_{100 \alpha(t)}$ indicam os valores correspondentes para cada curva na idade $t$.

Para criar os valores críticos para classificação do estado nutricional de acordo com o critério estatístico, foram utilizados na fórmula ${ }^{1}$ os valores $z$ equivalentes aos centis 85 e 95, recomendados para diagnosticar, respectivamente, excesso de peso e obesidade ${ }^{13,14}$. 
Para determinação dos valores críticos segundo o critério epidemiológico, foram fixados os valores finais desejados aos 20 anos: IMC igual a $25 \mathrm{~kg} / \mathrm{m}^{2}$ no caso do excesso de peso e IMC igual a $30 \mathrm{~kg} / \mathrm{m}^{2}$ no caso da obesidade. Aplicados na fórmula (2), esses valores permitirão estimar, retrospectivamente, seus valores equivalentes ao longo das idades anteriores.

$$
Z=\left[(I M C / M)^{L}-1\right] /(L S)
$$

No caso do baixo peso, haja vista o ponto de corte tradicionalmente recomendado $\left(18,5 \mathrm{~kg} / \mathrm{m}^{2}\right)$ não ter se mostrado adequado para essa classificação no padrão internacional ${ }^{5}$, foram realizadas análises adicionais a fim de se obter proposta que respondesse a essa necessidade. A seleção do valor crítico (aos 20 anos) para definir baixo peso levou em conta aspectos estatísticos e epidemiológicos. No caso do critério estatístico, procurou-se manter ao máximo a compatibilidade com aqueles critérios estatísticos usualmente praticados, ou seja, o valor de -2 escores $z$ para definição de déficits nutricionais. No caso do critério epidemiológico, buscou-se contemplar a plausibilidade do diagnóstico, observada segundo dois aspectos: 1) a prevalência esperada - informada pela densidade de freqüência do valor z - não pode ser igual ou superior àquela prevalência observada nas populações sob estudo, de modo a reduzir o número de casos falso-positivos e aprimorar a detecção dos verdadeiro-negativos; 2) foi selecionada a distribuição do IMC no início da fase adulta pertencente ao quarto superior da renda per capita, estrato social no qual é pouco provável haver déficit nutricional de natureza socioeconômica. Nesse grupo, o centil 3 do IMC entre 19,5 e 20,4 anos assumiu valores de $17,57 \mathrm{~kg} / \mathrm{m}^{2}$ no sexo masculino e $17,48 \mathrm{~kg} / \mathrm{m}^{2}$ no sexo feminino.

Aos 20 anos, na amostra de base populacional da PNSN, o valor de $z=-2(p=0,0228)$ equivale a valores de IMC de $17,78 \mathrm{~kg} / \mathrm{m}^{2}$ no sexo masculino e $17,14 \mathrm{~kg} / \mathrm{m}^{2}$ no sexo feminino.

Baseado nos pressupostos e evidências mencionados, selecionou-se, então, a curva correspondente ao IMC de $17,5 \mathrm{~kg} / \mathrm{m}^{2}$ (aos 20 anos) como ponto de corte para definir baixo peso na população brasileira. Assim, buscou-se conservar, também na classificação do baixo peso, o uso do valor único em ambos os sexos para classificação do estado nutricional de adultos.

Todas as análises foram realizadas com o pacote estatístico Stata (versão 8).

\section{Resultados}

O baixo percentual ( $1 \%$ ) de exclusão, seja por dados incompletos, seja por baixa plausibilidade biológica (IMC maior ou menor que 4 desvios padrão), é indicativo da consistência dos valores ao longo do espectro etário e evidência da qualidade das medidas antropométricas da base de dados utilizada, além de contribuir para preservar a representatividade nacional da amostra.
A subdivisão da amostra em grupos etários trimestrais propiciou número ( $\mathrm{n}$ ) médio de 190 casos (mínimo de 129 e máximo de 235) no sexo masculino e 183 (mínimo de 132 e máximo de 229) no sexo feminino.

Os valores dos parâmetros L, M e S para a população brasileira estão apresentados em intervalos semestrais segundo sexo na Tabela 1.

A suavização das curvas em cada sexo apresentou os seguintes resultados: a curva $L$ foi alisada com polinômio de $4^{\circ} \mathrm{grau}$ em ambos os sexos; a curva $M$, com polinômio de $4^{\circ}$ grau no sexo masculino e polinômio de $8^{\circ}$ grau no sexo feminino; a curva $\mathrm{S}$ foi ajustada com polinômio de $4^{\circ} \mathrm{grau}$ em ambos os sexos.

A Tabela 1 apresenta os parâmetros descritivos da distribuição de referência do IMC para a população brasileira entre 2 e 20 anos obtidos ao final do processo de modelagem. A Figura 1 apresenta, em cada sexo, sete centis da distribuição de referência.

Os valores $L$ permitem inferir a quantidade e o tipo de ajuste necessário para normalização do IMC no interior de cada estrato. A preponderância de valores negativos indica que os dados empregados na elaboração do padrão proposto apresentavam assimetria (skewness) à direita; a relativamente pequena amplitude do parâmetro $L$, em ambos os sexos, é indicativa da pequena magnitude da assimetria que era necessário remover. A média dos valores $L$ aproxima-se, nos dois sexos, de -1 . No intervalo etário em que se espera estejam contidos os momentos de pico do processo de maturação sexual (11 a 13 anos), o parâmetro $L$ apresenta valor médio de $-1,20$. O ponto mais intenso de remoção de assimetria situa-se na faixa etária entre 6 e 11 anos, momento anterior ao início do conjunto de fenômenos da maturação sexual, com valores $L$ de $-1,36$ no sexo masculino e $-1,38$ no sexo feminino.

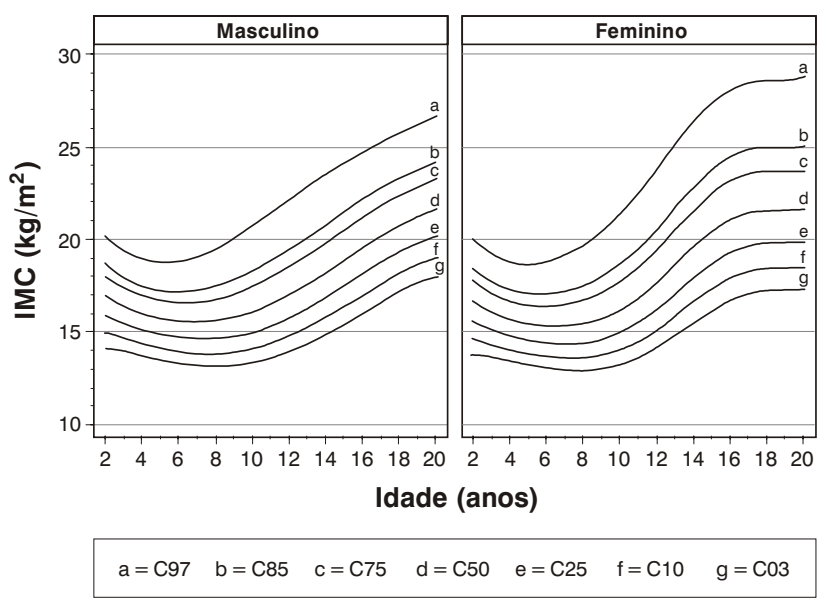

Figura 1 - Centis da distribuição da curva brasileira do IMC, em cada sexo

IMC = índice de massa corporal. 
A variabilidade média do IMC nos diferentes estratos, expressa pelo parâmetro S, foi cerca de $10 \%$ em cada sexo, com pequena amplitude (de 8 a 14\%). No intervalo etário no qual se espera esteja contido o ápice do processo de maturação sexual (11 a 13 anos), o parâmetro S apresenta média de $13,2 \%$.

A trajetória das curvas $\mathrm{S}$ ao longo da idade é, de início, declinante até próximo de 52 meses (4,3 anos). A partir dessa idade, assume trajetória ascendente até o período esperado da maturação sexual (11 a 13 anos). Nesse período, em ambos os sexos, os valores $\mathrm{S}$ atingem seus maiores valores. A seguir, ocorre período curto de estabilização em torno dos valores mais altos. Após a fase de maturação sexual, a curva $\mathrm{S}$ assume características diferenciadas segundo o sexo. No sexo masculino, a trajetória da curva S apresenta nítida redução da variabilidade, enquanto, no sexo feminino, a redução não é tão acentuada e a variabilidade permanece em torno de valores próximos àqueles do final da etapa de maturação sexual.

A Tabela 2 apresenta os valores críticos para classificação do baixo peso (curva equivalente ao IMC $17,5 \mathrm{~kg} / \mathrm{m}^{2}$ aos 20 anos), excesso de peso (curva equivalente ao IMC $25 \mathrm{~kg} / \mathrm{m}^{2}$ aos 20 anos) e obesidade (curva equivalente ao IMC $30 \mathrm{~kg} / \mathrm{m}^{2}$ aos 20 anos) para a população brasileira entre 2 e 20 anos obtidos a partir do emprego da fórmula (1).

Tabela 1 - Valores dos parâmetros L, M e S para a distribuição brasileira do índice de massa corporal na população de referência de 2 a 19 anos em cada sexo, segundo idade

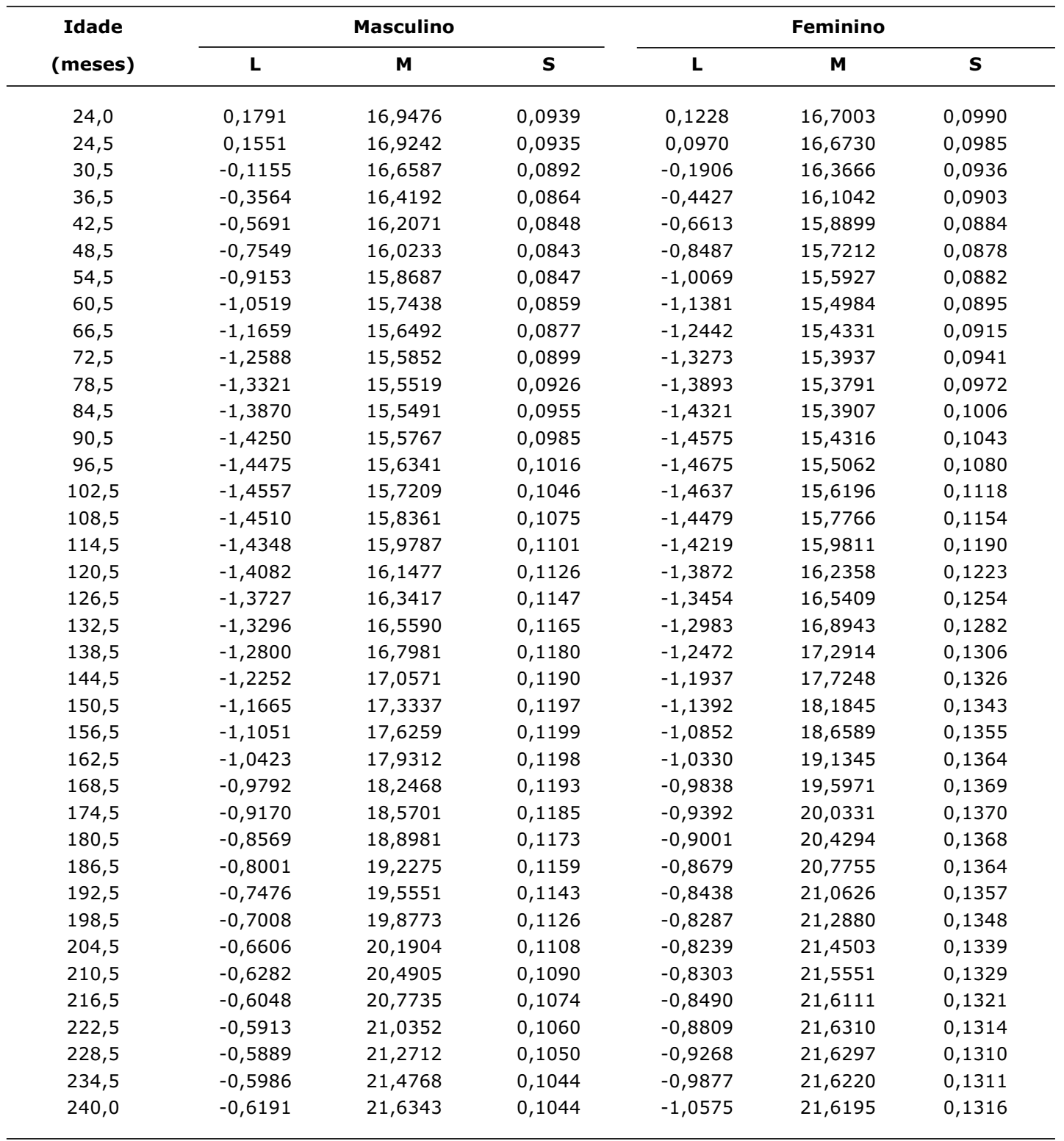


As curvas dos centis tradicionalmente utilizados como pontos de corte no caso do sobrepeso (centil 85) e da obesidade (centil 95) e as dos valores baseados em desfechos no início da vida adulta $\left(25 \mathrm{~kg} / \mathrm{m}^{2}\right.$ e $30 \mathrm{~kg} / \mathrm{m}^{2}$, respectivamente) estão expressas na Figura 2 . No sexo masculino, os valores das prevalências de baixo peso, sobrepeso e obesidade seriam superiores àqueles obtidos se, ao invés dos pontos de corte baseados nos valores dos adultos, fossem adotados os centis que tradicionalmente definem esses diagnósticos. No caso do sexo feminino, a mesma "superestimação" existiria apenas para o diagnóstico da obesidade; sobrepeso e baixo peso seriam classifi-

Tabela 2 - Valores críticos do IMC propostos para definição de baixo peso, excesso de peso e obesidade na população de referência brasileira de 2 a 19 anos em cada sexo, segundo idade

\begin{tabular}{|c|c|c|c|c|c|c|}
\hline \multirow{2}{*}{$\begin{array}{c}\text { Idade } \\
\text { (meses) }\end{array}$} & \multicolumn{3}{|c|}{ Masculino } & \multicolumn{3}{|c|}{ Feminino } \\
\hline & $\begin{array}{c}\text { BP } \\
\left(17,5 \mathrm{~kg} / \mathrm{m}^{2}\right)\end{array}$ & $\begin{array}{c}E P \\
\left(25 \mathrm{~kg} / \mathrm{m}^{2}\right)\end{array}$ & $\begin{array}{c}\text { OB } \\
\left(30 \mathrm{~kg} / \mathrm{m}^{2}\right)\end{array}$ & $\begin{array}{c}\text { BP } \\
\left(17,5 \mathrm{~kg} / \mathrm{m}^{2}\right)\end{array}$ & $\begin{array}{c}E P \\
\left(25 \mathrm{~kg} / \mathrm{m}^{2}\right)\end{array}$ & $\begin{array}{c}\text { OB } \\
\left(30 \mathrm{~kg} / \mathrm{m}^{2}\right)\end{array}$ \\
\hline 24,0 & 13,77 & 19,17 & 21,98 & 13,95 & 18,47 & 20,51 \\
\hline 24,5 & 13,77 & 19,13 & 21,94 & 13,94 & 18,43 & 20,47 \\
\hline 30,5 & 13,76 & 18,76 & 21,53 & 13,87 & 18,03 & 20,00 \\
\hline 36,5 & 13,70 & 18,45 & 21,21 & 13,76 & 17,70 & 19,64 \\
\hline 42,5 & 13,61 & 18,20 & 20,98 & 13,66 & 17,44 & 19,38 \\
\hline 48,5 & 13,50 & 18,00 & 20,85 & 13,55 & 17,26 & 19,22 \\
\hline 54,5 & 13,39 & 17,86 & 20,81 & 13,46 & 17,14 & 19,15 \\
\hline 60,5 & 13,28 & 17,77 & 20,85 & 13,37 & 17,07 & 19,16 \\
\hline 66,5 & 13,18 & 17,73 & 20,98 & 13,28 & 17,05 & 19,23 \\
\hline 72,5 & 13,09 & 17,73 & 21,19 & 13,21 & 17,07 & 19,37 \\
\hline 78,5 & 13,02 & 17,78 & 21,48 & 13,15 & 17,12 & 19,56 \\
\hline 84,5 & 12,96 & 17,87 & 21,83 & 13,10 & 17,20 & 19,81 \\
\hline 90,5 & 12,93 & 17,99 & 22,23 & 13,07 & 17,33 & 20,10 \\
\hline 96,5 & 12,91 & 18,16 & 22,69 & 13,07 & 17,49 & 20,44 \\
\hline 102,5 & 12,92 & 18,35 & 23,17 & 13,09 & 17,70 & 20,84 \\
\hline 108,5 & 12,95 & 18,57 & 23,67 & 13,16 & 17,96 & 21,28 \\
\hline 114,5 & 13,01 & 18,82 & 24,17 & 13,26 & 18,27 & 21,78 \\
\hline 120,5 & 13,09 & 19,09 & 24,67 & 13,40 & 18,63 & 22,32 \\
\hline 126,5 & 13,19 & 19,38 & 25,14 & 13,58 & 19,04 & 22,91 \\
\hline 132,5 & 13,32 & 19,68 & 25,58 & 13,81 & 19,51 & 23,54 \\
\hline 138,5 & 13,46 & 20,00 & 25,99 & 14,07 & 20,01 & 24,21 \\
\hline 144,5 & 13,63 & 20,32 & 26,36 & 14,37 & 20,55 & 24,89 \\
\hline 150,5 & 13,82 & 20,65 & 26,69 & 14,69 & 21,12 & 25,57 \\
\hline 156,5 & 14,02 & 20,99 & 26,99 & 15,03 & 21,69 & 26,25 \\
\hline 162,5 & 14,25 & 21,33 & 27,26 & 15,37 & 22,25 & 26,89 \\
\hline 168,5 & 14,49 & 21,66 & 27,51 & 15,72 & 22,79 & 27,50 \\
\hline 174,5 & 14,74 & 22,00 & 27,74 & 16,05 & 23,28 & 28,04 \\
\hline 180,5 & 15,01 & 22,33 & 27,95 & 16,35 & 23,73 & 28,51 \\
\hline 186,5 & 15,29 & 22,65 & 28,15 & 16,63 & 24,11 & 28,90 \\
\hline 192,5 & 15,58 & 22,96 & 28,34 & 16,87 & 24,41 & 29,20 \\
\hline 198,5 & 15,86 & 23,27 & 28,52 & 17,06 & 24,65 & 29,42 \\
\hline 204,5 & 16,15 & 23,56 & 28,71 & 17,22 & 24,81 & 29,56 \\
\hline 210,5 & 16,43 & 23,84 & 28,89 & 17,33 & 24,90 & 29,63 \\
\hline 216,5 & 16,70 & 24,11 & 29,08 & 17,40 & 24,95 & 29,67 \\
\hline 222,5 & 16,95 & 24,36 & 29,28 & 17,45 & 24,96 & 29,70 \\
\hline 228,5 & 17,18 & 24,59 & 29,50 & 17,47 & 24,96 & 29,74 \\
\hline 234,5 & 17,37 & 24,81 & 29,75 & 17,49 & 24,97 & 29,83 \\
\hline 240,0 & 17,50 & 25,00 & 30,00 & 17,50 & 25,00 & 30,00 \\
\hline Z & $-2,17$ & 1,32 & 2,83 & $-1,80$ & 1,02 & 2,10 \\
\hline $\mathrm{p}$ & 0,015 & 0,907 & 0,998 & 0,036 & 0,847 & 0,982 \\
\hline
\end{tabular}

$\mathrm{BP}=$ baixo peso; $\mathrm{EP}=$ excesso de peso; IMC = índice de massa corporal; $\mathrm{OB}=$ obesidade. 
cados de forma semelhante empregando-se os centis 85 e 3 ou os valores equivalentes a $25 \mathrm{~kg} / \mathrm{m}^{2}$ e $17,5 \mathrm{~kg} / \mathrm{m}^{2}$, respectivamente.

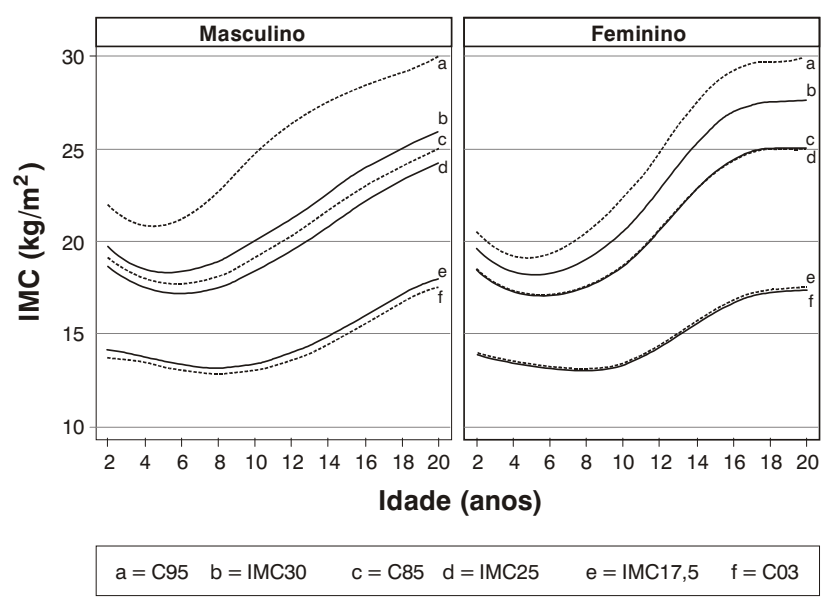

Figura 2 - Valores críticos (centis tradicionais e desfecho adulto) baseados na distribuição brasileira do índice de massa corporal para classificação do estado nutricional de crianças e adolescentes

IMC = índice de massa corporal.

\section{Discussão}

A discussão sobre as propriedades de um sistema nacional baseado no IMC para classificação do estado nutricional em crianças e adolescentes pode ser desdobrada na 1) apreciação da adequação da amostra utilizada na construção da referência e 2) na apreciação dos critérios selecionados para identificar os problemas nutricionais que se quer diagnosticar.

A análise do período 1974/1975 a 1989 e a análise do quarto mais rico da amostra da PNSN demonstram que a base de dados selecionada não foi afetada negativamente pelas tendências seculares do peso e da altura no grupo selecionado.

Dois outros trabalhos publicados, também baseados na PNSN, descrevem a distribuição do IMC segundo idade e sexo entre crianças e adolescentes brasileiros. O primeiro deles utilizou a faixa de 10 a 17 anos, em intervalos etários anuais. Os percentis 10 e 90 foram propostos como pontos de corte para o diagnóstico de déficit nutricional e sobrepeso, respectivamente. O percentil 90 foi escolhido por apresentar, aos 18 anos, valor coincidente com $25 \mathrm{~kg} / \mathrm{m}^{2}$. Os valores apresentados foram suavizados por média móvel${ }^{15}$. O segundo trabalho utilizou a faixa de 0 a 25 anos, em cada sexo. Os valores apresentados não foram submetidos a qualquer modelagem ${ }^{16}$.

Embora com diferenças no procedimento estatístico, os dois trabalhos apresentam valores semelhantes para a distribuição do IMC entre 10 e 17 anos, faixa etária comum aos dois trabalhos. A diferença essencial entre os dois trabalhos é que, enquanto Sichieri \& Allam ${ }^{15}$ geraram sua distribuição como uma referência nacional para a classificação nutricional segundo o IMC, Anjos et al. ${ }^{16}$ explicitamente recomendam que sua distribuição não seja utilizada para diagnóstico nutricional.

A modelagem de dados que se modificam com a idade e são, originalmente, assimétricos pode ser feita por diferentes métodos ${ }^{17}$. O uso do método LMS permitiu aplicar a transformação Box-Cox (eficiente na remoção de assimetria), bem como modelar independentemente o coeficiente de variação, mais do que do desvio padrão em si. Adicionalmente, o fato de o LMS ser o método mais amplamente utilizado no delineamento de curvas de referência do IMC permite maior comparabilidade internacional entre os parâmetros aqui construídos e aqueles de curvas de outros países ou internacionais. Alguns exercícios já realizados com esse intuito têm demonstrado que o uso da curva internacional pode alterar a prevalência do excesso de peso medida ao longo da infância e da adolescência. O sentido da alteração dependerá da faixa etária e das características do país ${ }^{18,19}$.

A decisão sobre qual critério adotar para a classificação do estado nutricional em crianças e adolescentes a partir do IMC é matéria ainda em aberto, embora mais recentemente esteja se firmando a opção pelo conjunto de curvas que retroagem os valores da classificação do estado nutricional entre adultos jovens para os indivíduos com idade abaixo de 18 ou 20 anos. No caso brasileiro, a opção pelo uso dos centis 85 e 95, ao invés daqueles baseados no desfecho do IMC adulto, implicaria obter, nas mulheres, valores mais altos para a prevalência de obesidade e, entre homens, obter valores mais altos para prevalência da desnutrição, do excesso de peso e da obesidade. A observação de crescimento na altura até próximo de 20 anos na população utilizada na construção da curva motivou a seleção dessa idade para o desfecho do IMC. A eventual opção pelo sistema classificatório baseado no desfecho em adultos deve, no entanto, ser realizada mediante debate mais amplo, no qual o aspecto da classificação do déficit nutricional deve ser tomado em conta.

A proposta aqui apresentada de utilizar o IMC igual a $17,5 \mathrm{~kg} / \mathrm{m}^{2}$ como valor crítico para classificação do déficit nutricional entre crianças e adolescentes requer, no entanto, análises adicionais e discussões mais amplas antes de sua eventual adoção.

O processo de maturação sexual, o qual cursa com modificações na composição corporal do indivíduo, pode tornar-se fator complicador para a avaliação nutricional baseada no IMC para idade. Assim, a análise das variabilidades entre e em cada geração torna-se questão-chave para a resposta à necessidade de controlar externamente ou não a fase de maturação sexual do indivíduo.

Evidências obtidas a partir de estudos populacionais apontam no sentido de a variabilidade intergeracional ser menor que a intrageracional. Entre elas: 1) a pequena diferença observada entre países quanto à idade mediana da menarca e a redução no ritmo da tendência secular, especialmente quando analisadas segundo as diferentes 
etapas propostas por Tanner20; 2) indícios de que, para o conjunto da população, países como EUA e Holanda não apresentam tendência à redução da idade da menarca no presente 21,22 ; 3) no Brasil, estima-se a redução na idade da menarca em 2,4 a 2,7 meses por década23,24.

Tomadas em seu conjunto, essas evidências permitem supor que o impacto da variabilidade intergeracional (incluídas as variabilidades intra e interindividuais) não deverá distorcer, no caso brasileiro, o uso da curva aqui apresentada.

\section{Referências}

1. Cole TJ. The use and construction of anthropometric growth reference standards. Nutr Res Rev. 1993;6:19-50.

2. Zerfas $A J$, Jelliffe DB, Jellife EFP. Epidemiology and nutrition. In: Falkner $F$, Tanner JM, editors. Human growth - a comprehensive treatise. New York: Plenum Press; 1986. p. 475-500.

3. Waterlow JC, Buzina R, Keller W, Lane JM, Nichaman MZ, Tanner JM. The presentation and use of height and weight data for comparing the nutritional status of groups of children under the age of 10 years. Bull World Health Organ. 1977;55:489-98.

4. Bellizzi MC, Dietz WH. Workshop on childhood obesity: summary of the discussion. Am J Clin Nutr. 1999;70:173S-5S.

5. Cole TJ, Bellizzi MC, Flegal KM, Dietz WH. Establishing a standard definition for child overweight and obesity worldwide: international survey. BMJ. 2000;320:1240-3.

6. Piers LS, Rowley KG, Soares MJ, O'Dea K. Relation of adiposity and body fat distribution to body mass index in Australians of Aboriginal and European ancestry. Eur J Clin Nutr. 2003;57: 956-63.

7. Reilly JJ. Assessment of childhood obesity: national reference data or international approach? Obes Res. 2002;10:838-40.

8. Cole TJ, Roede MJ. Centiles of body mass index for Dutch children aged 0-20 years in 1980 - a baseline to assess recent trends in obesity. Ann Hum Biol. 1999;26:303-8.

9. Wang $Y$. Is obesity associated with early sexual maturation? A comparison of the association in American boys versus girls. Pediatrics. 2002;110:903-10.

10. Bini V, Celi F, Berioli MG, Bacosi ML, Stella P, Giglio P, et al. Body mass index in children and adolescents according to age and pubertal stage. Eur J Clin Nutr. 2000;54:214-8.

11. Instituto Brasileiro de Geografia e Estatística, Fundo das Nações Unidas para a Infância. Perfil estatístico de crianças e mães no Brasil: aspectos de saúde e nutrição de crianças no Brasil. Rio de Janeiro: IBGE; 1992.
12. Cole TJ, Freeman JV, Preece MA. British 1990 growth reference centiles for weight, height, body mass index and head circumference fitted by maximum penalized likelihood. Stat Med. 1998; 17:407-29.

13. World Health Organization. Physical status: the use and interpretation of anthropometry. Geneva: WHO; 1995. WHO Technical Report Series no 854.

14. Barlow SE, Dietz WH. Management of child and adolescent obesity: summary and recommendations based on reports from pediatricians, pediatric nurse practitioners and registered dietitians. Pediatrics. 2002;110:236-8.

15. Sichieri R, Allam VLC. Avaliação do estado nutricional de adolescentes brasileiros através do índice de massa corporal. J Pediatr (Rio J). 1996;72:80-4.

16. Anjos LA, Veiga GV, Castro IRR. Distribuição dos valores do índice de massa corporal da população brasileira até 25 anos. Rev Panam Salud Publica. 1998;3:164-73.

17. Wright EM, Royston P. Simplified estimation of age-specific reference intervals for skewed data. Stat Med. 1997;16: 2785-803.

18. Wang $Y$, Wang JQ. A comparison of international references for the assessment of child and adolescent overweight and obesity in different populations. Eur J Clin Nutr. 2002;56:973-82.

19. Chinn S, Rona RJ. International definitions of overweight and obesity for children: a lasting solution? Ann Hum Biol. 2002;29:306-13.

20. Lee PA, Guo SS, Kulin HE. Age of puberty: data from the United States of America. APMIS. 2001;109:81-8.

21. Chumlea WC, Schubert CM, Roche AF, Kulin HE, Lee PA, Himes $\mathrm{JH}$, et al. Age at menarche and racial comparisons in US girls. Pediatrics. 2003;111:110-3.

22. Mul D, Fredriks AM, van Buuren S, Oostdijk W, VerlooveVanhorick SP, Wit JM. Pubertal development in the Netherlands 1965-1997. Pediatr Res. 2001;50:479-86.

23. Kac G, Auxiliadora de Santa Cruz Coel, Velasquez-Melendez G. Secular trend in age at menarche for women born between 1920 and 1979 in Rio de Janeiro, Brazil. Ann Hum Biol. 2000;27:423-8.

24. Junqueira Do Lago M, Faerstein $E$, De Souza Lopes $C$, Werneck GL; Pro-Saude Study (Rio de Janeiro, Brazil). Family socioeconomic background modified secular trends in age at menarche: evidence from the Pro-Saude Study (Rio de Janeiro, Brazil). Ann Hum Biol. 2003;30:347-52.

Correspondência:

Wolney Conde

Av. Dr. Arnaldo, 715, Departamento de Nutrição

CEP 01246-904 - São Paulo, SP

Tel./Fax: (11) 3066.7705

E-mail: wolney@usp.br 